hep-th/0003281

\title{
Hyperbolic Spaces in String and M-Theory
}

\author{
A. Kehagias ${ }^{1}$ and J.G. Russo ${ }^{2}$ \\ ${ }^{1}$ Physics Dept. National Technical University \\ GR-157 73 Zografou Campus, Athens, Greece \\ 2 Departamento de Física, Universidad de Buenos Aires \\ Ciudad Universitaria, Pabellón I, 1428 Buenos Aires \\ kehagias@mail.cern.ch, russo@df.uba.ar
}

\begin{abstract}
We describe string-theory and $d=11$ supergravity solutions involving symmetric spaces of constant negative curvature. Many examples of non-supersymmetric string compactifications on hyperbolic spaces $H_{r}$ of finite volume are given in terms of suitable cosets of the form $H_{r} / \Gamma$, where $\Gamma$ is a discrete group. We describe in some detail the cases of the non-compact hyperbolic spaces $F_{2}$ and $F_{3}$, representing the fundamental regions of $H_{2}$ and $H_{3}$ under $S L(2, Z)$ and the Picard group, respectively. By writing $A d S$ as a $U(1)$ fibration, we obtain new solutions where $A d S_{2 p+1}$ gets untwisted by T-duality to $\mathbf{R} \times S U(p, 1) /(S U(p) \times U(1))$. Solutions with time-dependent dilaton field are also constructed by starting with a solution with NS5-brane flux over $H_{3}$. A new class of non-supersymmetric conformal field theories can be defined via holography.
\end{abstract}

March 2000 


\section{Introduction}

Maximally symmetric spaces play an important role in supergravity [1] and in superstring theory [2], and they naturally arise as the near-horizon region of black brane geometries [3, 4. Maximally symmetric spaces (with no more than one time dimension) are the de Sitter $d S_{p}$ and anti de Sitter $A d S_{p}$ spaces, $n$-spheres $S^{n}$ and $r$-dimensional hyperboloids $H_{r}$. Although supergravity solutions describing spheres and $A d S$ spaces have been extensively investigated [1], not much has been done for solutions involving the de Sitter and hyperbolic geometries. There are several obvious reasons for this. De Sitter spaces always break supersymmetry, and they do not describe universes with zero cosmological constant. Hyperbolic spaces, such as the upper half $r$-plane, admit Killing spinors [5, 6, 7] but they have infinite volume. Therefore, they do not seem useful for describing internal spaces in string compactifications. Finite volume examples can be constructed by cosets of the form $H_{r} / \Gamma$, where $\Gamma$ is a discrete subgroup of the isometry group of $H_{r}$ [8, 9, 10]. However, dividing by $\Gamma$ breaks all supersymmetries (see sect. 3 ).

We shall show that there is a large class of new string compactifications involving $H_{r}$ or cosets $H_{r} / \Gamma$, which are regular (away possible orbifold points), where $\alpha^{\prime}$ string corrections can be made very small for a sufficiently large radius of $H_{r}$. Among the possible applications of these solutions, we explore: a) the definition of new $\mathcal{N}=0$ conformal field theories in four dimensions via holography (see also [11, 12]); b) obtaining new models for dimensional reduction in non-compact spaces.

This paper is organized as follows. In sect. 2, we describe solutions of elevendimensional supergravity representing spaces which are direct products containing $H_{r}$ factors and discuss the supersymmetry. Analogous solutions in type II supergravity are also given. In sect. 3, we discuss finite-volume compactifications. In sect. 4 we compute the Newton gravitational potential for spaces having the hyperbolic 3-manifold $H_{3}$ as internal space. Because the Kaluza-Klein eigenvalue spectrum on $\mathrm{H}_{3}$ is continuous with a mass gap, the Newton potential has a Yukawa-type behavior at large distances. Hyperbolic spaces naturally appear in theories with other spacetime signatures 13, 14, 15 (e.g. by Wick rotating $A d S$ solutions to Euclidean space) or in solutions with imaginary gauge fields, which can be interpreted as solutions with real gauge fields in new type II* theories [14, where the signs of kinetic terms of RR gauge fields are reversed. In sect. 5, we consider two different approaches for obtaining new solutions in these theories. One of them consists in writing $A d S_{2 p+1}$ as a $U(1)$ fibration and performing

T-duality in a time-like direction. In sect. 6, we discuss the dual conformal field theories in compactifications that are direct product of anti de Sitter, hyperbolic manifolds of finite volume and spheres. Finally, we calculate in the appendix the Euler number of the space $S U(2,1) / S U(2) \times U(1)$.

\section{Hyperbolic manifolds in Supergravity}




\subsection{Eleven-dimensional Supergravity}

The eleven-dimensional graviton multiplet contains the graviton $g_{M N}$, the antisymmetric three-form $A_{M N K}$ and the gravitino $\Psi_{M}(M, N, \ldots=0, \ldots, 10)$. The bosonic part of the eleven-dimensional supergravity Lagrangian is

$$
\mathcal{L}=\frac{1}{2 \kappa_{11}^{2}} \sqrt{g}\left(R-\frac{1}{2 \cdot 4 !} F_{M N P Q} F^{M N P Q}\right)-\frac{1}{12^{4}} \epsilon^{M_{1} \ldots M_{11}} A_{M_{1} M_{2} M_{3}} F_{M_{4} \ldots M_{7}} F_{M_{8} \ldots M_{11}} .
$$

A well-known solution to the equations of motions

$$
\begin{aligned}
R_{M N} & =\frac{1}{12}\left(F_{M P Q R} F_{N}^{P Q R}-\frac{1}{12} g_{M N} F^{2}\right), \\
\nabla_{M} F^{M N P Q} & =-\frac{1}{1152} \epsilon^{N P Q R_{1} \ldots R_{8}} F_{R_{1} \ldots R_{4}} F_{R_{5} \ldots R_{8}},
\end{aligned}
$$

is provided by the Freund-Rubin ansatz for the antisymmetric field strength

$$
\begin{aligned}
F_{m n p q} & =6 m_{0} \epsilon_{m n p q} \text { for } m, n, \ldots=7, \ldots, 10 \\
F_{M N P Q} & =0, \quad \text { otherwise. }
\end{aligned}
$$

By substituting this ansatz into the field equations (2) we get

$$
\begin{aligned}
R_{\mu \nu} & =-6 m_{0}^{2} g_{\mu \nu}, \quad \mu, \nu=0, \ldots 6, \\
R_{m n} & =12 m_{0}^{2} g_{m n} .
\end{aligned}
$$

The requirement of unbroken supersymmetry, i.e., the vanishing of the gravitino transformation

$$
\delta \Psi_{M}=\nabla_{M} \epsilon-\frac{1}{288}\left(\Gamma_{M}^{P Q R S}-8 \delta_{M}^{P} \Gamma^{Q R S}\right) \epsilon F_{P Q R S},
$$

for the ansatz (国), is equivalent to the existence of $S O(1,6)$ and $S O(4)$ Killing spinors $\theta$ and $\eta$, respectively, which satisfy

$$
\begin{aligned}
\nabla_{\mu} \theta & = \pm \frac{1}{2} m_{0} \gamma_{\mu} \theta, \\
\nabla_{m} \eta & = \pm m_{0} \gamma_{m} \eta,
\end{aligned}
$$

where $\gamma_{\mu}\left(\gamma_{m}\right)$ are $S O(1,6)(S O(4)) \gamma$-matrices. Thus, although eq. (5) is solved for $Y^{7} \times$ $X^{4}$ where $Y^{7}$ and $X^{4}$ are Einstein spaces of negative and positive curvature, respectively, only those spaces that admit Killing spinors obeying eqs. (7), (8) are supersymmetric. The integrability condition of eqs.(17), (8) are

$$
W_{\mu \nu \rho \sigma} \gamma^{\rho \sigma} \theta=0, \quad W_{m n p q} \gamma^{p q} \eta=0,
$$

where $W_{\mu \nu \rho \sigma}, W_{m n p q}$ are the Weyl tensors of $Y^{7}, X^{4}$, respectively. Thus, obvious supersymmetric examples for $X^{4}$ include the round four-sphere $S^{4}$ and its orbifolds $S^{4} / \Gamma$, where $\Gamma$ is an appropriate discrete group [16]. For the $Y^{7}$ space one can take the anti-de 
Sitter space $A d S_{7}$, which preserves supersymmetry as well, and leads to the $A d S_{7} \times S^{4}$ vacuum of eleven-dimensional supergravity.

In addition to solutions of the form $A d S_{7} \times X^{4}$, there are other solutions to eq. (可) involving hyperbolic manifolds. The spaces $A d S_{7-r} \times H_{r} \times S^{4}$, where $H_{r}, r \geq 2$ is the $r$-dimensional upper-half space with metric:

$$
d s^{2}=d H_{r}^{2} \equiv \frac{R_{0}^{2}}{x_{0}^{2}}\left(d x_{0}^{2}+d x_{1}^{2}+\ldots+d x_{r}^{2}\right), \quad R_{0}^{2}=\frac{r}{6 m_{0}^{2}},
$$

solve eqs. (2), (3). Thus, the following backgrounds are all vacua of eleven-dimensional supergravity:

i) $A d S_{2} \times H_{5} \times S^{4}$;

ii) $\mathrm{Ad} \mathrm{S}_{2} \times \mathrm{H}_{2} \times \mathrm{H}_{3} \times \mathrm{S}^{4}$;

iii) $\mathrm{AdS}_{3} \times \mathrm{H}_{2} \times \mathrm{H}_{2} \times \mathrm{S}^{4}$;

iv) $A d S_{3} \times H_{4} \times S^{4}$;

v) $A d S_{4} \times H_{3} \times S^{4}$;

vi) $A d S_{5} \times H_{2} \times S^{4}$.

Neither of these backgrounds are supersymmetric. Indeed, they do not satisfy eq. (9). [Note that the product-space of conformally flat spaces is not conformally flat]. However, although supersymmetry automatically guarantees the stability of the background, its absence does not necessarily implies instability. We recall for example the cases of $A d S_{4} \times$ $M^{p q r}$ [18] and $A d S_{4} \times Q^{p q r}$ [19] compactifications of eleven-dimensional supergravity, which are stable for a certain range of $p, q$ [20, 21]. A general result of the stability analysis in [20] was that, a generic $A d S_{4} \times M_{7}$ compactification, where the Einstein space is a product of an $n$-dimensional manifold $M_{(1)}^{n}$ and a $7-n$-dimensional one $M_{(2)}^{7-n}$, $M_{7}=M_{(1)}^{n} \times M_{(2)}^{7-n}$, is unstable. This is due to the fact that the stability of the $A d S_{4}$ vacuum depends on the eigenvalues of the Lichnerowicz operator $\Delta_{L}$ in the internal space $M_{7}$ acting on transverse, tracefree symmetric tensors $h_{m n}$. The background is supersymmetric if $\Delta_{L}$ is strictly positive (in fact $\Delta_{L}$ should be bigger than a multiple of $\left.m_{0}^{2}\right)$. However, for product spaces, $\Delta_{L}$ has a zero eigenvalue with eigenfunction $h_{m n}=$ $\operatorname{diag}\left(g_{m_{1} n_{1}},-\frac{n}{(7-n)} g_{m_{2} n_{2}}\right)$, where $g_{m_{1} n_{1}}, g_{m_{2} n_{2}}$ are the metrics on $M_{(1)}^{n}, M_{(2)}^{7-n}$, respectively. Clearly, this eigenfunction corresponds to an expanding $M_{(1)}^{n}$ and a contracting $M_{(2)}^{7-n}$ with constant total volume of $M_{(1)}^{n} \times M_{(2)}^{7-n}$. Thus, the instability is due to volume-preserving metric deformations of the internal space.

All the (i)-(vi) vacua are clearly of the form described above, so it may appear that they are unstable if $H_{r}$ is replaced by a finite volume coset $H_{r} / \Gamma$, as it will be described in the next section. However, this is not the case. Let us consider for example the case (v) involving $A d S_{4}$. Fixing the $A d S$ radius, the corresponding radius of $H_{r}$ is also fixed and instabilities can only be produced by metric perturbations on $S^{4}$ which preserve the Einstein metric. Such perturbations do not exist and although the background is of product form, it is stable. Of course, a definite statement for the stability of (i)-(vi) needs 
a case-by-case study of the spectrum. However, as the latter has not been studied yet, at least the potentially dangerous volume-preserving metric deformations of the internal space are absent.

Other non-supersymmetric compactifications can be obtained by replacing $H_{r}$ in the spaces i) - vi) by any Einstein space of the same negative curvature. In particular, one has the solution

vii) $A d S_{3} \times \Sigma_{4} \times S^{4}$

where $\Sigma_{4}$ is the coset space $S U(2,1) /(S U(2) \times U(1))$. This space will appear again in sect. 5.1 (see also the appendix).

\subsection{Hyperbolic manifolds in type II supergravity}

Simple type IIB supergravity backgrounds with D3 brane charge and constant dilaton are the following ones:

i) $A d S_{3} \times H_{2} \times S^{5}$;

ii) $A d S_{2} \times H_{3} \times S^{5}$.

Like $A d S_{5} \times S^{2} \times S^{3}$, these spaces have no Killing spinors. In particular, in the case ii), the metric is given by

$$
d s_{10 B}^{2}=\frac{R^{2}}{4 z^{2}}\left(-d t^{2}+d z^{2}\right)+\frac{R^{2}}{2 v^{2}}\left(d x^{2}+d y^{2}+d v^{2}\right)+R^{2} d \Omega_{5}^{2} .
$$

The 5-form self-dual field strength is the same as in the $A d S_{5} \times S^{5}$ solution. The isometry group is $S O(1,2) \times S O(1,3) \times S O(6)$. Although the solution is not supersymmetric, it is regular everywhere (being a direct product of Einstein spaces), and $\alpha^{\prime}$ corrections can be made very small for sufficiently large radius $R$.

\section{Finite volume cosets $H_{r} / \Gamma$}

In the previous section we have seen some supergravity solutions involving factors $A d S_{p}$ and $H_{r}$. The space $H_{r}$ has infinite volume (with respect to the Poincaré metric). Thus there are no normalizable modes for any field in $H_{r}$, and the bulk as well as the boundary theories are both empty. Non-empty bulk and boundary field theories can be obtained by forming the coset $H_{r} / \Gamma$ where $\Gamma$ is a discrete subgroup of the isometry group of the upper-half plane. We shall now discuss some specific examples.

\section{$3.1 \quad$ Killing spinors}

The supergravity solutions involving the $H_{r}$ manifolds discussed in sect. 2 are not supersymmetric. However, our analysis does not exclude that there could be more complicated 
backgrounds involving $H_{r}$, where some supersymmetries are unbroken. The Poincaré manifolds $H_{r}$ admit Killing spinors (explicit expressions can be found in [6]), and a question of general interest is whether they survive under the orbifolding by the discrete group $\Gamma$. For this we may describe the upper-half space $H_{r}$ as the hypersurface (taken for simplicity to be of constant curvature -1 )

$$
-X_{0}^{2}+X_{1}^{2}+\ldots+X_{r}^{2}=-1 .
$$

The isometry group of $H_{r}$ is then clearly $S O(1, r)$ and the group $\Gamma$ is in general a subgroup of $S O(1, r)$, which may or may not have fixed points. There are two cases to be discussed. If $r=e v e n$, then a Killing spinor is in the spinorial representation of $S O(1, r-1)$. Then, if $\Gamma$ is a subgroup of $S O(1, r-1)$, but it is not a subgroup of $S O(1, r-3)$, then there are no surviving Killing spinors. The latter exist if $\Gamma \subset S O(1, r-3)$. However, in this case $H_{r} / \Gamma$ will still be of infinite volume. Thus there are no finite volume cosets $H_{r} / \Gamma$, with $r=e v e n$, with unbroken supersymmetries. In particular, any supergravity compactification involving finite volume cosets $H_{2} / \Gamma$ and $H_{4} / \Gamma$ will not preserve any supersymmetry.

The second case is when $r=o d d$. In this case, there are two Killing spinors on $H_{r}$ in the spinorial representation of $S O(1, r-1)$. These two Killing spinors are also Weyl spinors of the isometry group $S O(1, r)$, so that they form an irreducible Dirac spinor of $S O(1, r)$. If $\Gamma$ is not a subgroup $S O(1, r-1)$, then all supersymmetries are broken, while if $\Gamma$ is a subgroup $S O(1, r-1)$, then half of the supersymmetries survive. In this case, it may seem that the space $H_{r} / \Gamma$ can be of finite volume. In the particular case of $H_{3}$, we will explicitly see below that there is no choice of $\Gamma$ that lead to a finite volume supersymmetric space.

\subsection{Solutions of the form $A d S^{5} \times H_{2} / \Gamma \times S^{4}$}

Let us consider in first place the $A d S^{5} \times H_{2} / \Gamma \times S^{4}$ solution of eleven-dimensional supergravity. The metric and four-form field strength are given by

$$
\begin{gathered}
d s_{11}^{2}=\frac{2 R_{0}^{2}}{3 z^{2}}\left(-d t^{2}+d x_{1}^{2}+d x_{2}^{2}+d x_{3}^{2}+d z^{2}\right)+\frac{R_{0}^{2}}{6 v^{2}}\left(d x^{2}+d v^{2}\right)+\frac{R_{0}^{2}}{4} d \Omega_{4}^{2}, \\
\hat{F}_{4}=6 R_{0}^{3} \Omega_{4},
\end{gathered}
$$

where $d \Omega_{4}^{2}$ is the metric on $S^{4}$ and we have taken the Poincaré metric for the upper half-plane $H_{2}(v>0)$. A finite volume hyperbolic space can be obtained by forming the coset $H_{2} / \Gamma$, where $\Gamma$ is a discrete subgroup of the isometry group $S L(2, \mathbf{R})$ of the upperhalf plane. Thus, $\Gamma$ must be a Fuchsian group, and the requirement of finiteness of the volume of $H_{2} / \Gamma$ is equivalent to the Fuchsian group $\Gamma$ to be of the first kind. Examples are compact hyperbolic manifolds representing genus 2 Riemann surfaces.

\footnotetext{
${ }^{1}$ The $r=$ odd case may be compared to the coset $S^{2 n+1} / \Gamma$ [17, 11, 12]. $S^{5} / \Gamma$ is supersymmetric if $\Gamma \subset S U(3), S U(2)$, while is not supersymmetric if $\Gamma$ is a maximal subgroup of $S U(4)$.
} 
A non-compact space of finite volume can be constructed by taking $\Gamma=S L(2, \mathbf{Z})$, so that $H_{2} / \Gamma$ is the fundamental domain $F_{2}$ of $S L(2, \mathbf{Z})$. This case provides a rather simple setup for dimensional reduction, since there are no Kaluza-Klein modes associated with non-trivial quantum numbers in $F_{2}$. Modular functions which are eigenstates of the Laplacian cannot be square-integrable, excepting the constant mode. Indeed, for a square-integrable wave function obeying $\square \psi=\lambda \psi$, one can drop a boundary term in the following integration by parts

$$
0 \leq \int_{F_{2}} d \mu \partial \psi^{*} \partial \psi=-\int_{F_{2}} d \mu \psi^{*} \square \psi=-\lambda \int_{F_{2}} d \mu \psi^{*} \psi, \quad d \mu=\frac{d v d x}{v^{2}},
$$

which implies $\lambda \leq 0$. But this contradicts the fact that the Laplace operator is negative in the fundamental domain (i.e. all eigenfunctions have $\lambda \geq 0$ ). Thus, scalar modes in $A d S_{5} \times F_{2} \times S^{4}$ will be of the form $\psi=\phi(A d S) Y\left(S^{4}\right)$, with no dependence on the $F_{2}$ coordinates.

\subsection{The $A d S_{4} \times F_{3} \times S^{4}$ model}

Let us now consider the $A d S_{4} \times H_{3} / \Gamma \times S^{4}$ background. Here the supergravity solution is given by

$$
\begin{gathered}
d s_{11}^{2}=\frac{R_{0}^{2}}{2 z^{2}}\left(-d t^{2}+d x_{1}^{2}+d x_{2}^{2}+d z^{2}\right)+\frac{R_{0}^{2}}{3 v^{2}}\left(d x^{2}+d y^{2}+d v^{2}\right)+\frac{R_{0}^{2}}{4} d \Omega_{4}^{2}, \\
\hat{F}_{4}=6 R_{0}^{3} \Omega_{4},
\end{gathered}
$$

In ref. [9], it was considered the case when $H_{3} / \Gamma$ is the hyperbolic dodecahedron space, which is a compact hyperbolic manifold obtained by identifying opposite faces of a dodecahedron after a $3 \pi / 5$ rotation. More generally, we will show below that any finite volume space of the form $H_{3} / \Gamma$ does not admit Killing spinors. We will mainly be interested in the case when $\mathrm{H}_{3}$ is a non-compact hyperbolic manifold given by the fundamental region of the Picard group, which we describe next.

The space $H_{3}$ finds its natural description in terms of the quaternionic upper half 3 -space (further details can be found in [22]). To define it, it is useful to recall a few known facts about quaternions, which are objects of the form

$$
q=x+i y+j u+k v,
$$

with $x, y, u, v$ real, where

$$
i j=k=-j i, \quad j k=i=-k j, \quad k i=j=-i k, \quad i^{2}=j^{2}=k^{2}=-1
$$

The norm of a quaternion is given by $\|q\|=\sqrt{x^{2}+y^{2}+u^{2}+v^{2}}$. Quaternions can be represented in terms of Pauli matrices, so that $S U(2)$ can be viewed as the unit quaternion. 
The quaternionic upper half 3-plane is defined as follows:

$$
H_{3}=\{x+i y+k v \mid x, y, v \in \mathbf{R}, v>0\}
$$

Thus the elements of $H_{3}$ are quaternions with $j$-coordinate equal to zero. It can be identified with the symmetric space $S L(2, \mathbf{C}) / S U(2)$. A matrix $g \in S L(2, \mathbf{C})$ acts on an element $q$ of the quaternionic upper half plane in the following way:

$$
q^{\prime} \equiv g(q)=(a q+b)(c q+d)^{-1}, \quad g=\left(\begin{array}{ll}
a & b \\
c & d
\end{array}\right)
$$

with $v^{\prime}=v\|c q+d\|^{-2}$. The metric, volume element, and Laplacian on $H_{3}$ are given by

$$
\begin{aligned}
& d s^{2}=\left(d x^{2}+d y^{2}+d v^{2}\right) v^{-2} \\
& d \mu=v^{-3} d x d y d v \\
& \Delta=v^{2}\left(\partial^{2} / \partial x^{2}+\partial^{2} / \partial y^{2}+\partial^{2} / \partial v^{2}\right)-v \partial / \partial v .
\end{aligned}
$$

The Picard group is defined to be

$$
\Gamma=S L(2, \mathbf{Z}[i])=\left\{g=\left(\begin{array}{ll}
a & b \\
c & d
\end{array}\right) \mid a, b, c, d \in \mathbf{Z}[i], \operatorname{det} g=1\right\},
$$

with

$$
\mathbf{Z}[i]=\{x+i y \mid x, y \in \mathbf{Z}\}
$$

A fundamental domain for $S L(2, \mathbf{Z}[i]) \backslash H_{3}$ in the quaternionic upper half plane is then

$$
F_{3}=\left\{x+i y+k v|| x \mid \leq 1 / 2,0 \leq y \leq 1 / 2, x^{2}+y^{2}+v^{2} \geq 1, v>0\right\} .
$$

The volume of the fundamental domain $F_{3}$ is 22

$$
\operatorname{Vol}\left(F_{3}\right)=\int_{F_{3}} d \mu=\frac{|D|^{3 / 2} \zeta_{K}(2)}{4 \pi^{2}},
$$

where $\zeta_{K}(2)$ is the Dedekind zeta function of the number field $\mathbf{Q}(i)$.

Although non-supersymmetric, the space $A d S_{4} \times F_{3} \times S^{4}$ is an interesting setup for string compactification, in particular, for the construction of conformal field theories in $2+1$ dimensions (see sect. 6).

One can show that there is no supersymmetric compactification in supergravity involving $F_{3}$. Indeed, it is easy to see that no Killing spinor survives after dividing $H_{3}$ by $S L(2, \mathbf{Z}[i])$. The isometry group of $H_{3}$ is $S O(1,3) \cong S L(2, \mathbf{R}) \times S L(2, \mathbf{R})$. There are two Killing spinors which are Weyl spinors of each $S L(2, \mathbf{R})$ factor. The Picard group $S L(2, \mathbf{Z}[i])$ is essentially given by the subgroup $S L(2, \mathbf{Z}) \times S L(2, \mathbf{Z})$ of $S L(2, \mathbf{R}) \times S L(2, \mathbf{R})$. Therefore it does not leave invariant any of the Killing spinors. More generally, any finite volume example of the form $H_{3} / \Gamma$ will not have Killing spinors, since the finite-volume condition implies that $\Gamma$ must contain elements of both factors in $S L(2, \mathbf{R}) \times S L(2, \mathbf{R})$. 


\section{Newton's law on $H_{3}$}

Having described a number of string compactifications containing hyperbolic spaces, a question of interest (in view of the recent results of [23] for Newton's law in certain noncompact spaces) is whether such compactifications may be compatible with the observed Newton's law. In the case of compactification on a compact space $X$, there exist massive Kaluza-Klein modes of the four-dimensional graviton associated with the eigenvalues of the Laplace operator on $X$. In the static limit, these modes contribute to the Newton's law giving, in addition to the standard $1 / r$ behavior of the massless graviton, Yukawatype corrections. In particular, these are the expected corrections if the internal space is a compact hyperbolic manifold of the form $H_{r} / \Gamma$. What is the form of the corrections if the internal space is the non-compact, infinite volume hyperbolic manifold $H_{r}$ ? For noncompact spaces, the spectrum of the Laplace operator is continuous. For a continuum spectrum of eigenvalues, the gravitational potential is given by [24]

$$
V(r) \sim \frac{1}{r} f(r)
$$

where

$$
f(r)=\int d q e^{-q r}\left|\Psi_{q}(0)\right|^{2}
$$

and

$$
-\nabla^{2} \Psi_{q}(\rho)=q^{2} \Psi_{q}(\rho)
$$

The standard $1 / r$ behavior can still be recovered in some non-compact spaces [23].

Let us consider for simplicity $M^{4} \times H_{3}$, where $M^{4}$ is the four-dimensional Minkowski space. Then, in "spherical" coordinates $(\rho, \theta, \phi)$ for $H_{3}$ and metric

$$
d s^{2}\left(H_{3}\right)=d \rho^{2}+\sinh ^{2} \rho\left(d \theta^{2}+\sin ^{2} \theta d \phi^{2}\right),
$$

the eigenmodes are 25

$$
\Psi_{q \ell m}(\rho, \theta, \phi)=R_{q}^{\ell}(\rho) Y_{\ell m}(\theta, \phi),
$$

with a continuous spectrum of eigenvalues $q^{2}=[1, \infty)$. The functions $Y_{\ell m}$ are spherical harmonics, and the radial eigenfunctions are the hyperspherical Bessel functions

$$
R_{q}^{\ell}(\rho)=\frac{(-1)^{\ell+1} \sinh ^{\ell} \rho}{\left(\prod_{n=0}^{\ell}\left(n^{2}+k^{2}\right)\right)^{1 / 2}} \frac{d^{\ell+1} \cos (k \rho)}{d(\cosh \rho)^{\ell+1}}, \quad k^{2}=q^{2}-1
$$

In the $\ell=0$ sector (i.e. spherical modes with radial dependence only), we obtain

$$
f(r)=\int_{1}^{\infty} d q \sqrt{q^{2}-1} e^{-q r}=\frac{K_{1}(r)}{r},
$$

where $K_{1}(r)$ is the modified Bessel function of the second kind. The gravitational potential in four dimensions is then

$$
V(r) \sim \frac{K_{1}(r)}{r^{2}}
$$


At distances $r$ much smaller than the radius of the hyperboloid (here taken to be 1 for simplicity), one has $V(r) \sim 1 / r^{3}$. At large distances $r \gg 1$, the potential falls off

exponentially, $V(r)=\sqrt{\frac{\pi}{2 r^{5}}} e^{-r}$. This is expected, since there is a gap in the spectrum. As there is no any local minimum of $K_{1}(r) / r$, a $1 / r$ approximated behavior cannot be obtained at any scale.

\section{Hyperbolic spaces in type IIA*/IIB* supergravi- ties}

\section{1 $A d S_{2 p+1}$ as a $U(1)$ fibration and untwisting}

In ref. [26], a type IIA solution of the form $A d S_{5} \times C P_{2} \times S^{1}$ was obtained by starting with $A d S_{5} \times S^{5}$, writing the 5 -sphere as a $\mathrm{U}(1)$ bundle over $C P_{2}$ (Hopf fibration) and performing T-duality along $S^{1}$. Now we make an analogous formal construction of a new type IIA solution containing a hyperbolic space, by starting with a type IIB solution describing $A d S_{3}$ or $A d S_{5}$, and performing a similar operation on the $A d S$ part of the space. One can write $A d S_{2 n+1}$ as a $U(1)$ bundle over a $2 n$-dimensional base space, and then perform T-duality in the time direction, which untwists the fibration. As pointed out in [14, T-duality in a time-like direction does not connect type IIA/IIB theory, but rather relates type IIA to a type IIB* and type IIB to type IIA*, obtained by reversing the signs of RR kinetic terms.

Let us illustrate this by first considering the case of the $A d S_{3} \times S^{3} \times T^{4}$ vacuum of type IIB superstring theory, representing the geometry of the near-horizon D1-D5 brane system. The $A d S_{3}$ space is the hyperboloid

$$
-X_{0}^{2}+X_{1}^{2}+X_{2}^{2}-X_{3}^{2}=-R^{2}
$$

or,

$$
-W \bar{W}+Z \bar{Z}=-1 \quad Z=X_{1}+i X_{2}, \quad W=X_{0}+i X_{3} .
$$

For simplicity, we have set the radius equal to one. The extension to an arbitrary radius is straightforward. By writing

$$
Z=\frac{R}{\left(\frac{r^{2}}{R^{2}}-1\right)^{1 / 2}} e^{i t}, \quad W=\frac{r}{\left(\frac{r^{2}}{R^{2}}-1\right)^{1 / 2}} e^{i(t+\varphi)},
$$

one obtains the $A d S_{3}$ metric in the following form

$$
d s^{2}=\frac{d r^{2}+r^{2} d \varphi^{2}}{\left(1-\frac{r^{2}}{R^{2}}\right)^{2}}-R^{2}\left(d t+\frac{r^{2}}{R^{2}-r^{2}} d \varphi\right)^{2} .
$$

The existence of a $U(1)$ symmetry and a $U(1)$ fibration over a base $H_{2}$ is manifest in these coordinates. The fibration is trivial and can be turned into a non-trivial one after dividing $\mathrm{H}_{2}$ by a discrete $\Gamma \subset S L(2, \mathbf{R})$. The form of the metric eq. (32) makes also 
manifest the appearance of closed time-like curves. These can be avoided by unwrapping the time direction or, equivalently, by considering the universal covering space of $A d S_{3}$, which does not have any closed time-like curve. For the purpose of obtaining a new solution of type II supergravity, we can formally perform a T-duality transformation along the $S^{1}$ compactified time direction in eq. (32). By using the standard T-duality transformation rules [27], we get the dual solution

$$
d \hat{s}^{2}=\frac{d r^{2}}{\left(1-\frac{r^{2}}{R^{2}}\right)^{2}}+\frac{r^{2}}{\left(1-\frac{r^{2}}{R^{2}}\right)^{2}} d \varphi^{2}-R^{2} d t^{2}, \quad B_{t \varphi}=-\frac{r^{2}}{1-\frac{r^{2}}{R^{2}}}, \quad e^{2 \phi}=g^{2}=\text { const. . }
$$

There are also components of the 2-form and 4-form field strength tensors given by

$$
\hat{F}_{2}=\frac{R^{2} r}{\left(1-\frac{r^{2}}{R^{2}}\right)^{2}} d r \wedge d \varphi, \quad \hat{F}_{4}=2 R^{2} d t \wedge \Omega_{3}
$$

Remarkably enough, the metric

$$
d \sigma^{2}=\frac{d r^{2}}{\left(1-\frac{r^{2}}{R^{2}}\right)^{2}}+\frac{r^{2}}{\left(1-\frac{r^{2}}{R^{2}}\right)^{2}} d \phi^{2}
$$

is the metric of the upper-half plane $H_{2}$. Thus, the T-dual of the $A d S_{3} \times S^{3}$ vacuum is the space $\mathbf{R} \times H_{2} \times S^{3}$, where we have unwrapped the time direction.

Let us now consider the anti de Sitter space $A d S_{5}$. In terms of complex coordinates, it is the hyperboloid:

$$
-W \bar{W}+Z_{1} \bar{Z}_{1}+Z_{2} \bar{Z}_{2}=-R^{2}
$$

Write

$$
\begin{gathered}
W=\frac{R e^{i t}}{\left(1-\frac{r^{2}}{R^{2}}\right)^{1 / 2}}, \quad Z_{1}=\frac{r}{\left(1-\frac{r^{2}}{R^{2}}\right)^{1 / 2}} e^{i\left(t+\frac{\chi+\varphi}{2}\right)} \sin \frac{\theta}{2}, \\
Z_{2}=\frac{r}{\left(1-\frac{r^{2}}{R^{2}}\right)^{1 / 2}} e^{i\left(t+\frac{\chi-\varphi}{2}\right)} \cos \frac{\theta}{2} .
\end{gathered}
$$

The metric becomes

$$
d s^{2}\left(A d S_{5}\right)=d \Sigma_{4}^{2}-R^{2}(d t-A)^{2},
$$

where

$$
\begin{gathered}
d \Sigma_{4}^{2}=\frac{d r^{2}}{\left(1-\frac{r^{2}}{R^{2}}\right)^{2}}+\frac{1}{4} \frac{r^{2}}{\left(1-\frac{r^{2}}{R^{2}}\right)}\left(d \theta^{2}+\sin ^{2} \theta d \varphi^{2}\right)+\frac{1}{4} \frac{r^{2}}{\left(1-\frac{r^{2}}{R^{2}}\right)^{2}}(d \chi-\cos \theta d \varphi)^{2}, \\
A=\frac{r^{2}}{2\left(R^{2}-r^{2}\right)}(d \chi-\cos \theta d \varphi) .
\end{gathered}
$$

Now we make T-duality in time direction. This gives a new type IIA* supergravity solution. After T-duality, we get

$$
d s_{10 A}^{2}=d \Sigma_{4}^{2}-R^{2} d t^{2}+R^{2} d \Omega_{5}^{2}, \quad B_{2}=R^{2} d t \wedge A, \quad e^{2 \phi}=g^{2} .
$$

There is also the 4 -form field strength $d A_{3}$ with components in $\Sigma_{4}$ implied by the Tduality rules. Thus $A d S_{5} \times S^{5}$ gets untwisted to a space $\mathbf{R} \times \Sigma_{4} \times S^{5}$. The space $\Sigma^{4}$ has 
self-dual Weyl tensor and, in fact, it is the constant negative-curvature partner of $C P_{2}$, with Ricci tensor

$$
\mathcal{R}_{\mu \nu}\left(\Sigma_{4}\right)=-\frac{6}{R^{2}} g_{\mu \nu}\left(\Sigma_{4}\right) .
$$

Indeed, after $r \rightarrow i r$, we find that $d \Sigma_{4}^{2} \rightarrow-d s^{2}\left(C P_{2}\right)$. It is not difficult to see that the space $\Sigma_{4}$ is the coset space $S U(2,1) / S U(2) \times U(1)$. In general, the $A d S_{2 n+1}$ can be untwisted to $\Sigma_{2 n} \times S^{1}$ where $\Sigma_{2 n}$ is the constant negative-curvature partner of $C P_{n}$ with metric obtained from the latter after $r \rightarrow i r$ in the Fubini-Study metric of $C P_{n} . \Sigma_{2 n}$ is in fact the coset space $S U(n, 1) / S U(n) \times U(1)$. In the appendix we calculate the Euler number of this space.

\subsection{Solutions with fluxes on $H_{r}$}

A conformal field theory involving the upper half 3 -space $H_{3}$ can be constructed as a WZW model based on the coset $S L(2, \mathbf{C}) / S U(2)$. This can be combined with another CFT by direct product. In particular, it can be combined with the NS5-brane by summing both conformal sigma models (similar constructions have been done in [28]). To

saturate the central charge, one needs to add a linear dilaton in the time direction. The construction leads to an NS-NS 2-form gauge field with imaginary components. By Sduality, one can convert it into a R-R 2-form with imaginary components, and thus into a solution of type IIB* theory. The resulting conformal model is an exact solution of string theory to all orders in the $\alpha^{\prime}$-expansion.

These sigma models can also be constructed directly by brane intersections (the basic rules for constructing intersecting branes are in [29]). We first describe simpler string solutions based on the embedding $H_{3}$ in $\mathbf{R}^{10}$ (the extension to other $H_{r}$ is straightforward). Let us consider spaces of the form $X_{4} \times M_{6}$, with metric:

$$
d s^{2}=-d t^{2}+t^{2} d H_{3}^{2}+d s_{6}^{2}, \quad d H_{3}^{2}=\frac{1}{z^{2}}\left(d x^{2}+d y^{2}+d z^{2}\right),
$$

where $d s_{6}^{2}$ describes the six-dimensional compact manifold $M_{6}$. The hyperbolic 3-manifold $H_{3}$ arises as constant time slices of $X_{4}$. The Riemann tensor for $X_{4}$ vanishes identically. To see this, one introduces new coordinates

$$
U=t / z, \quad X=t x / z, \quad Y=t y / z, \quad V=t z+\frac{t}{z}\left(x^{2}+y^{2}\right)
$$

so that the metric for $X_{4}$ takes the form

$$
d s_{4}^{2}=-d U d V+d X^{2}+d Y^{2}
$$

Thus eq. (41) provides the embedding of $H_{3}$ in a four-dimensional space-time. If $M_{6}=T^{6}$, then the metric (40) is an exact solution of string theory, since the Riemann tensor identically vanishes. To leading order in $\alpha^{\prime}$, one can take any Ricci-flat space $M_{6}$. From eq. (41) one gets

$$
t^{2}=U V-X^{2}-Y^{2} .
$$


The condition that $t^{2} \geq 0$ implies that the physical space-time is in the region $U V \geq$ $X^{2}+Y^{2}$.

To construct solutions with brane charges, a natural starting point is the NS5 brane, with flux on $H_{3}$. It is given by

$$
\begin{gathered}
d s_{10}^{2}=d x_{i}^{2}+f(t)\left(-d t^{2}+t^{2} d H_{3}^{2}\right), \quad d H_{3}^{2}=\frac{1}{z^{2}}\left(d x^{2}+d y^{2}+d z^{2}\right), \quad i=1, \ldots, 6 \\
e^{2 \phi}=f(t), \quad i d B=* d f, \quad f(t)=1+\frac{R^{2}}{t^{2}} .
\end{gathered}
$$

This is a formal analog of the NS five brane solution, in which the 3-sphere has been replaced by a hyperbolic space. Note that the gauge field $d B$ is imaginary. By S-duality, and redefining the RR 2 -form by a factor $i$, this is converted into a solution of type IIB* supergravity.

To have finite flux, the space $H_{3}$ can be replaced by the finite volume $F_{3}$ space, representing the fundamental region of the Picard group, as described previously (or by any coset $H_{3} / \Gamma$ of finite volume).

By an appropriate rescaling of variables, one finds the "near horizon" solution:

$$
d s_{10}^{2}=d x_{i}^{2}-d \tau^{2}+R^{2} d H_{3}^{2}, \quad \phi=R^{-1} \tau, \quad \tau=-\ln t .
$$

This is a direct product of flat space with linear dilaton and a $S L(2, \mathbf{C}) / S U(2)$ WZW model.

We now generalize the solution (44) by considering the intersection with an NS5 brane

$$
\begin{aligned}
& d s_{10}^{2}=d x_{1}^{2}+d x_{2}^{2}+f(t)\left(-d t^{2}+t^{2} d H_{3}^{2}\right)+g(r)\left(d r^{2}+r^{2} d \Omega_{3}^{2}\right), \\
& e^{2 \phi}=f(t) g(r), \quad i d B=* d f(t)+* d g(r), \quad g(r)=1+\frac{R_{0}^{2}}{r^{2}} .
\end{aligned}
$$

Similarly, the NS5-brane can be combined with the solution (46), giving

$$
\begin{gathered}
d s_{10}^{2}=-d \tau^{2}+d x_{1}^{2}+d x_{2}^{2}+R^{2} d H_{3}^{2}+g(r)\left(d r^{2}+r^{2} d \Omega_{3}^{2}\right) \\
e^{2 \phi}=e^{\frac{2 \tau}{R}} g(r) .
\end{gathered}
$$

One can also add a fundamental string in the direction $x_{1}$ as follows:

$$
\begin{gathered}
d s_{10}^{2}=h^{-1}(r)\left[-d \tau^{2}+d x_{1}^{2}\right]+d x_{2}^{2}+R^{2} d H_{3}^{2}+g(r)\left(d r^{2}+r^{2} d \Omega_{3}^{2}\right), \\
e^{2 \phi}=e^{\frac{2 \tau}{R}} \frac{g(r)}{h(r)}, \quad h(r)=1+\frac{R_{1}^{2}}{r^{2}} .
\end{gathered}
$$

The near-horizon geometry is given by

$$
\begin{gathered}
d s_{10}^{2}=\frac{r^{2}}{R_{1}^{2}}\left[-d \tau^{2}+d x_{1}^{2}\right]+R_{0}^{2} \frac{d r^{2}}{r^{2}}+R^{2} d H_{3}^{2}+R_{0}^{2} d \Omega_{3}^{2} \\
e^{\phi}=e^{\frac{\tau}{R}} \frac{R_{0}}{R_{1}} .
\end{gathered}
$$


This describes a background $A d S_{3} \times S^{3} \times H_{3} \times S^{1}$, with a linear dilaton in the time direction.

By U-duality, one can construct different D-brane solutions with time dependence. In particular, starting with (44) and making $\mathrm{S}$ and T-dualities, one obtains solutions with $\mathrm{D} p$ brane charge, with $p>3$ :

$$
\begin{gathered}
d s_{10 B}^{2}=f^{-1 / 2}(t)\left(d x_{1}^{2}+\ldots+d x_{p+1}^{2}\right)+f^{-1 / 2}(t)\left[-d t^{2}+t^{2} d H_{8-p}^{2}\right] \\
e^{2 \phi}=f^{(3-p) / 2}, \quad \hat{F}_{8-p}=i R^{7-p} \hat{H}_{8-p}, \quad f=1+\frac{R^{7-p}}{t^{7-p}},
\end{gathered}
$$

where $\hat{H}_{r}$ is the volume form of $H_{r}$. In the case of $p=3$, one gets near $t=0$ a direct product of de Sitter space-time and the hyperboloid $H_{5}$, i.e. $d S_{5} \times H_{5}$ (which can also be obtained from the $S^{5} \times A d S_{5}$ solution by Wick rotation).

\section{Discussion}

The backgrounds described in sect. 2 can be used for the construction of new $\mathcal{N}=0$ conformal field theories by holography. A version of the Maldacena dualities states that string/M theory on any space of the form $A d S_{d+1} \times X$ is equivalent to a conformal field theory in $d$ dimensions. In particular, in the case of the $A d S_{7} \times S^{4}$ compactification, the boundary theory is a CFT for the six-dimensional $\mathcal{N}=2$ tensor multiplet [2], or a CFT for the six-dimensional $\mathcal{N}=1$ vector multiplet in the $A d S_{7} \times S^{4} / \Gamma$ background [16]. According to this, string (or M) theory compactified on spaces of the form $A d S_{d+1} \times\left(H_{r} / \Gamma\right) \times S^{n}$ defines a $d$ dimensional conformal field theory with $S O(n+1)$ global symmetry. The correlation functions of the CFT are defined by the prescription [30, 31]

$$
\left\langle e^{\int d^{d} x \phi_{0}(x) \mathcal{O}(x)}\right\rangle_{\mathrm{CFT}} \equiv Z_{\text {string }}\left(\phi(\text { boundary })=\phi_{0}(x)\right),
$$

where $Z_{\text {string }}$ is the string theory partition function computed with boundary values $\phi_{0}(x)$ of the string theory fields, which act as sources of CFT operators. As usual, in the classical supergravity approximation, it can be evaluated as $Z_{\text {string }} \cong \exp \left[-I_{S G}(\phi)\right]$, by solving the equations of motion of $\phi$ in the background for $A d S_{d+1} \times\left(H_{r} / \Gamma\right) \times S^{n}$, with the boundary condition $\phi$ (boundary) $=\phi_{0}(x)$. Consider, in particular, the $d=11$ supergravity solution $A d S_{5} \times F_{2} \times S^{4}$. This should be dual to a $d=4$ non-supersymmetric CFT with $S O(5)$ global symmetry group. Because the supergravity solution has M5-brane charge, one may expect the CFT to be related to the six-dimensional $(2,0)$ or $(1,0)$ conformal field theories. As explained in sect. 3, eigenfunctions of the Laplace operator on $F_{2}$ are not square-integrable, barring the constant mode. Thus Kaluza-Klein modes obeying the Laplace equation are constant in $F_{2}$. In the absence of supersymmetry, finding the field theory degrees of freedom is nevertheless difficult because the field theory is expected to be strongly coupled in the regime the supergravity description is valid.

Other interesting cases are the eleven-dimensional supergravity solution $A d S_{4} \times F_{3} \times$ $S^{4}$, and the type IIB solution $A d S_{3} \times F_{2} \times S^{5}$. Since the latter has D3 brane charge, 
it should be dual to some $\mathcal{N}=0 d=1+1$ conformal field theory related to $\mathcal{N}=4$

$d=3+1$ super Yang-Mills theory. The $A d S_{4} \times F_{3} \times S^{4}$ solution has M5 brane charge. The dual field theory should be a $d=2+1$ conformal field theory related to the $6 d(2,0)$ CFT, with an internal global symmetry group given by $S O(5)$.

\section{Acknowledgements}

We would like to thank A. Tseytlin for useful comments, and S. Randjbar-Daemi for an important remark. A.K. is supported by a Г.Г.E.T. grant No. 97/E $/ 71$. J.R. would like to thank the support of UBA, Conicet and Fundación Antorchas. 


\section{APPENDIX}

In sect. 5 we have seen that the anti de Sitter spacetime $A d S_{2 p+1}$ can be represented as a $U(1)$ fibration over a base space $\Sigma_{2 p}=S U(p, 1) /(S U(p) \times U(1))$. Here we present the calculation of the Euler number of $\Sigma_{4}$, which we expect will be of use for future applications. The space $\Sigma_{4}$ is a homogeneous space of the group $S U(2,1)$ and it is diffeomorphic to $S U(2,1) / S U(2) \times U(1)$. Its metric is given in eq. (37) and by using the standard $S U(2)$ left-invariant one-forms $\sigma_{i}, i=1,2,3, \quad\left(d \sigma_{1}=2 \sigma_{2} \wedge \sigma_{3}, \ldots\right)$ on the $S^{3}$, we may express eq. (37) as

$$
d \Sigma_{4}^{2}=\frac{d r^{2}+r^{2} \sigma_{3}^{2}}{\left(1-r^{2}\right)^{2}}+\frac{r^{2}\left(\sigma_{1}^{2}+\sigma_{2}^{2}\right)}{\left(1-r^{2}\right)}
$$

where we have put $R=1$. Recalling that the metric on $C P_{2}$ is

$$
d C P_{2}^{2}=\frac{d r^{2}+r^{2} \sigma_{3}^{2}}{\left(1+r^{2}\right)^{2}}+\frac{r^{2}\left(\sigma_{1}^{2}+\sigma_{2}^{2}\right)}{\left(1+r^{2}\right)}
$$

we see that the above metrics eqs. $(58,59)$ on $\Sigma_{4}=S U(2,1) / S U(2) \times U(1)$ and $C P_{2}=$ $S U(3) / S U(2) \times U(1)$ are related by $r \rightarrow i r$. Note that although $C P_{2}$ is compact, $\Sigma_{4}$ is not and it has a boundary at $r=1$.

The Euler number $\chi\left(\Sigma_{4}\right)$ is calculated by the formula

$$
\chi(M)=\frac{1}{32 \pi^{2}} \int_{M} \epsilon^{a b c d} R_{a b} \wedge R_{c d}-\frac{1}{32 \pi^{2}} \int_{\partial M} \epsilon^{a b c d}\left(2 \theta_{a b} \wedge R_{c d}-\frac{4}{3} \theta_{a b} \wedge \theta_{c e} \wedge \theta_{e d}\right) .
$$

The first term is the standard bulk contribution whereas the last term is for the boundary corrections. A straightforward calculation for $\Sigma_{4}$ gives

$$
\chi\left(\Sigma_{4}\right)=3\left(1-\frac{1-2 r_{0}}{\left(1-r_{0}^{2}\right)^{2}}\right)-\frac{3 r_{0}^{2}+2 r_{0}^{4}}{\left(1-r_{0}^{2}\right)^{2}}+\frac{1+2 r_{0}^{2}}{\left(1-r_{0}^{2}\right)^{2}}=1 .
$$

On the other hand, the Euler number of $C P_{2}$ is $\chi\left(C P_{2}\right)=3$ so the Euler numbers of $C P_{2}$ and $\Sigma_{2}$ differ by two units. This difference is due to the bold singularity that exists in the $C P_{2}$ space and it is absence in the case of $\Sigma_{4}$. Indeed, the $C P_{2}$ metric for $r>>1$ turns out to be

$$
d C P_{2}^{2} \sim \rho^{-4}\left(d r^{2}+r^{2} \sigma_{3}^{2}\right)+\sigma_{1}^{2}+\sigma_{2}^{2},
$$

and the surface $r \rightarrow \infty$ is a removable bolt singularity. The space $\Sigma$ does not have any bolt. Since each bolt contributes two units to the Euler number, the Euler number of $C P_{2}$ will exceed the corresponding one of $\Sigma_{4}$ by two, as indeed happens.

\section{References}

[1] M. J. Duff, B. E. Nilsson and C. N. Pope, Phys. Rept. 130, 1 (1986).

[2] J. Maldacena, Adv. Theor. Math. Phys. 2 (1998) 231, hep-th/9711200. 
[3] G. T. Horowitz and A. Strominger, Nucl. Phys. B360, 197 (1991).

[4] G.W. Gibbons and P.K. Townsend, Phys. Rev. Lett. 71 (1993) 3754.

[5] P. Breitenlohner and D. Z. Freedman, Annals Phys. 144, 249 (1982).

[6] Y. Fujii and K. Yamagishi, J. Math Phys. 27 (1986) 979.

[7] H. Lu, C. N. Pope and P. Townsend, Phys. Lett. B391, 39 (1997), hep - th/9607164;

H. Lu, C. N. Pope and J. Rahmfeld, J. Math. Phys. 40, 4518 (1999), hep-th/9805151.

[8] J. Milnor, Bull. Math. Soc. 6 (1982) 9.

[9] I.Ya. Aref'eva and I.V. Volovich, Nucl.Phys. B274 (1986) 619.

[10] N. Kaloper, J. March-Russell, G. D. Starkman and M. Trodden, "Compact hyperbolic extra dimensions: Branes, Kaluza-Klein modes and cosmology", hepph/0002001.

[11] S. Kachru and E. Silverstein, Phys. Rev. Lett. 80, 4855 (1998), hep-th/9802183.

[12] A. Lawrence, N. Nekrasov and C. Vafa, Nucl. Phys. B533, 199 (1998), hepth/9803015.

[13] E. Cremmer, I. V. Lavrinenko, H. Lu, C. N. Pope, K. S. Stelle and T. A. Tran, "Euclidean-signature supergravities, dualities and instantons," Nucl. Phys. B534, 40 (1998), hep-th/9803259.

[14] C. M. Hull, "Timelike T-duality, de Sitter space, large N gauge theories and topological field theory," JHEP 9807, 021 (1998), hep-th/9806146.

[15] C. M. Hull and R. R. Khuri, "Worldvolume theories, holography, duality and time," hep-th/9911082.

[16] S. Ferrara, A. Kehagias, H. Partouche and A. Zaffaroni, Phys. Lett. B431, 42 (1998), hep-th/9803109; Phys. Lett. B431, 57 (1998) hep-th/9804006.

[17] M. R. Douglas and B. R. Greene, Adv. Theor. Math. Phys. 1, 184 (1998) hepth/9707214; M. R. Douglas, B. R. Greene and D. R. Morrison, Nucl. Phys. B506, 84 (1997) hep-th/9704151.

[18] E. Witten, Nucl. Phys. B186, 412 (1981);

L. Castellani, R. D'Auria and P. Fre, Nucl. Phys. B239, 610 (1984).

[19] R. D'Auria, P. Fre and P. van Nieuwenhuizen, Phys. Lett. B136, 347 (1984).

[20] M. J. Duff, B. E. Nilsson and C. N. Pope, Phys. Lett. B139, 154 (1984).

[21] D. N. Page and C. N. Pope, Phys. Lett. B144, 346 (1984); Phys. Lett. B145, 333 (1984). 
[22] A. Terras, "Harmonics analysis on symmetric spaces and applications," I, II, Springer-Verlag 1985.

[23] L. Randall and R. Sundrum, Phys. Rev. Lett. 83, 4690 (1999), hep-th/9906064.

[24] A. Kehagias and K. Sfetsos, Phys. Lett. B427 39 (2000), hep-ph/9905417; A. Kehagias, hep-th/9911134.

[25] N.J. Cornish and D.N. Spergel, "On the eigenmodes of compact hyperbolic 3manifolds," math.DG/9906017.

[26] M. J. Duff, H. Lu and C. N. Pope, Nucl. Phys. B532, 181 (1998), hep-th/9803061.

[27] E. Bergshoeff, C. Hull and T. Ortín, Nucl. Phys. B451, 547 (1995), hep-th/9504081.

[28] G. Papadopoulos, J. G. Russo and A. A. Tseytlin, "Curved branes from string dualities," Class. Quant. Grav. 17, 1713 (2000), hep-th/9911253.

[29] A.A. Tseytlin, Class. Quant. Grav. 14 (1997) 2085; hep-th/9702163.

[30] S. S. Gubser, I. R. Klebanov and A. M. Polyakov, "Gauge theory correlators from non-critical string theory," Phys. Lett. B428, 105 (1998), hep-th/9802109.

[31] E. Witten, Adv. Theor. Math. Phys. 2 (1998) 253, hep-th/9802150. 